\title{
Vibration-Rotation Bands of Carbonyl Sulfide
}

\author{
Arthur G. Maki, Earle K. Plyler, and Eugene D. Tidwell
}

(December 8, 1961)

\begin{abstract}
Thirty-two infrared absorption bands of carbonyl sulfide (OCS) have been measured and are reported here, seventeen for the first time. Seven absorption bands due to isotopic molecules were also measured. Rotational constants have been determined and the evaluation of the anharmonicity constants is discussed.
\end{abstract}

\section{Introduction}

Although considerable previous work has been done on the infrared spectrum of OCS [1-3], ${ }^{1}$ too few absorption bands had been measured to allow a determination of all the anharmonicity constants of this molecule. This work was undertaken to measure enough bands to enable such a determination to be made. When combined with previously published work, the additional bands reported here should be sufficient to determine the quadratic anharmonicity constants provided the Fermi resonance between $\nu_{1}$ and $2 \nu_{2}$ is the only vibrational perturbation of appreciable size. However, when the constants were calculated, it was found that some of the constants had varying values depending on the bands used in their determination. This discrepancy will be discussed in a later section.

\section{Experimental Procedures}

The spectra were measured on the NBS high-resolution spectrometer which has a $5 \times 8$ in. grating with 10,000 lines/in. The optical arrangement of the spectrometer was designed so that the grating could be used double pass for maximum resolution. With favorable conditions, such as low pressure, the spectrometer will partially resolve lines separated by $0.03 \mathrm{~cm}^{-1}$.

The high resolution was made possible by using a cooled PbS cell as detector. Further details concerning the instrument which was designed and built at the Bureau have been given in a previous publication [4].

The high resolution measurements at wavelengths greater than $4.5 \mu$ were obtained using a 7,500 lines/in. grating and a cooled PbSe detector.

A multiple reflection gas cell with a path length of $8 \mathrm{~m}$ was used for the measurements. Various pressures were used although whenever possible pressures of a few $\mathrm{mm}$ were employed so that the maximum resolution could be attained. Some variation of the gas temperature was also utilized. Temperatures of

1 Figures in brackets indicate the literature references at the end of this paper.
$60{ }^{\circ} \mathrm{C}$ were sometimes used to aid in identification of lines due to "hot bands."

The spectra were measured by using the fringe system of a Fabry-Perot interferometer as a comparison spectrum. The fringe system and absorption spectrum were recorded simultaneously by the use of a double pen recorder [5]. All wavelengths measured were reduced to wavenumbers in vacuum.

The carbonyl sulfide gas was obtained from the Matheson Company. It contained impurities of $\mathrm{CS}_{2}, \mathrm{CO}_{2}$, and $\mathrm{HCN}$. It was purified by Roland Florin of the Bureau and the mass spectrometer analysis of the final product showed it to be about 99.8 percent pure. There were very small amounts of $\mathrm{CS}_{2}(0.2 \%)$ and $\mathrm{HCN}(0.03 \%)$ in the sample.

Thirty-two bands were measured in the spectral range from 1,890 to $6,100 \mathrm{~cm}^{-1}$. Some of these bands had been measured by previous workers at lower resolution, but those bands were remeasured in the attempt to obtain a more consistent set of energy levels. The 001-000 transition was measured by Callomon and Thompson [1]. They reported the band center at $2062.22 \mathrm{~cm}^{-1}$. In the present work the value obtained was $2062.22 \mathrm{~cm}^{-1}$ which is in excellent agreement. Likewise, the agreement is very good between the value of 4101.387 $\mathrm{cm}^{-1}$ obtained in this work for $2 \nu_{3}$ and the value $4101.384 \mathrm{~cm}^{-1}$ obtained by Saksena, Wiggins, and Rank (see ref. 3).

Since the average spacing of the rotational lines in the bands of OCS is about $0.4 \mathrm{~cm}^{-1}$, the overlapping required very good resolution to reveal the true structure and especially the l-type doubling. In figures 1, 2, and 3 are shown the types of bands observed in three regions of the spectrum. In figure 1 is shown on a slow scan (two $\mathrm{cm}^{-1}$ per foot of record) the $Q$ branch and part of the $P$ branch through P10 of the $01^{1} 1-000$ transition. Overlapping these two branches is the $R$ branch of the $300-000$ transition. Figures 2 and 3 are records obtained at a much faster scanning rate $(1 \mathrm{ft}$ of record per $15 \mathrm{~cm}^{-1}$ ). In figure 3 a number of lines arising from water vapor of the atmosphere are superimposed on the spectrum of OCS. These water vapor lines have been identified and marked with a small circle on the figure. 


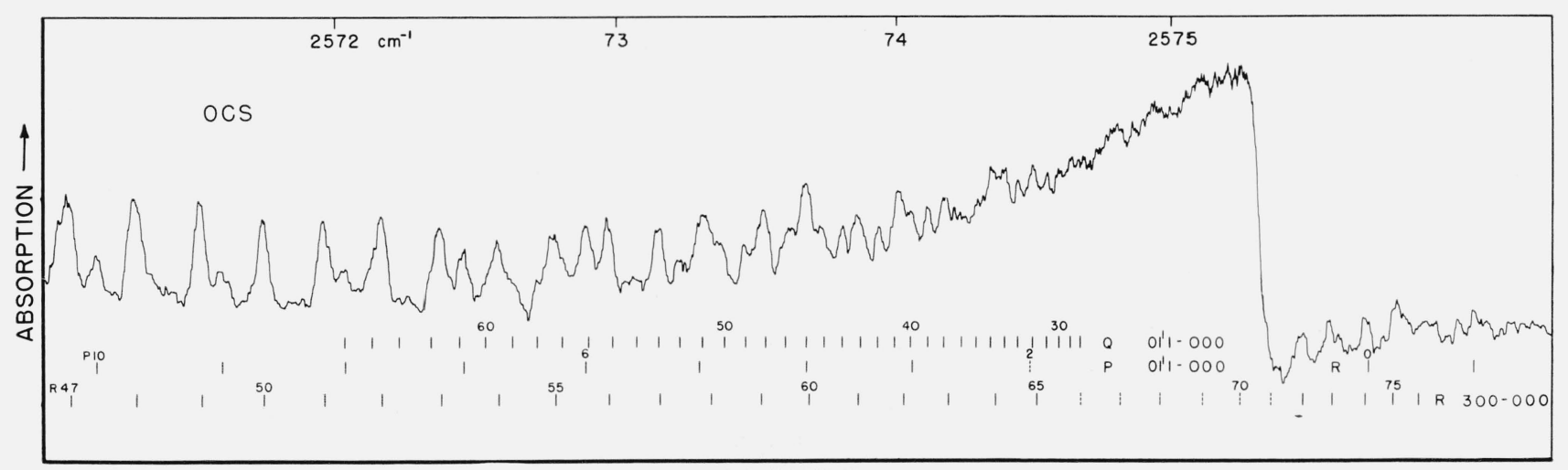

WAVENUMBER $\longrightarrow$

Figure 1. The Q branch of $\nu_{2}^{1}+\nu_{3}$ at $25 \% 5.32 \mathrm{~cm}^{-1}$.

The pressure was $3 \mathrm{~cm}$ of $\mathrm{Hg}$ with a path of $8 \mathrm{~m}$.

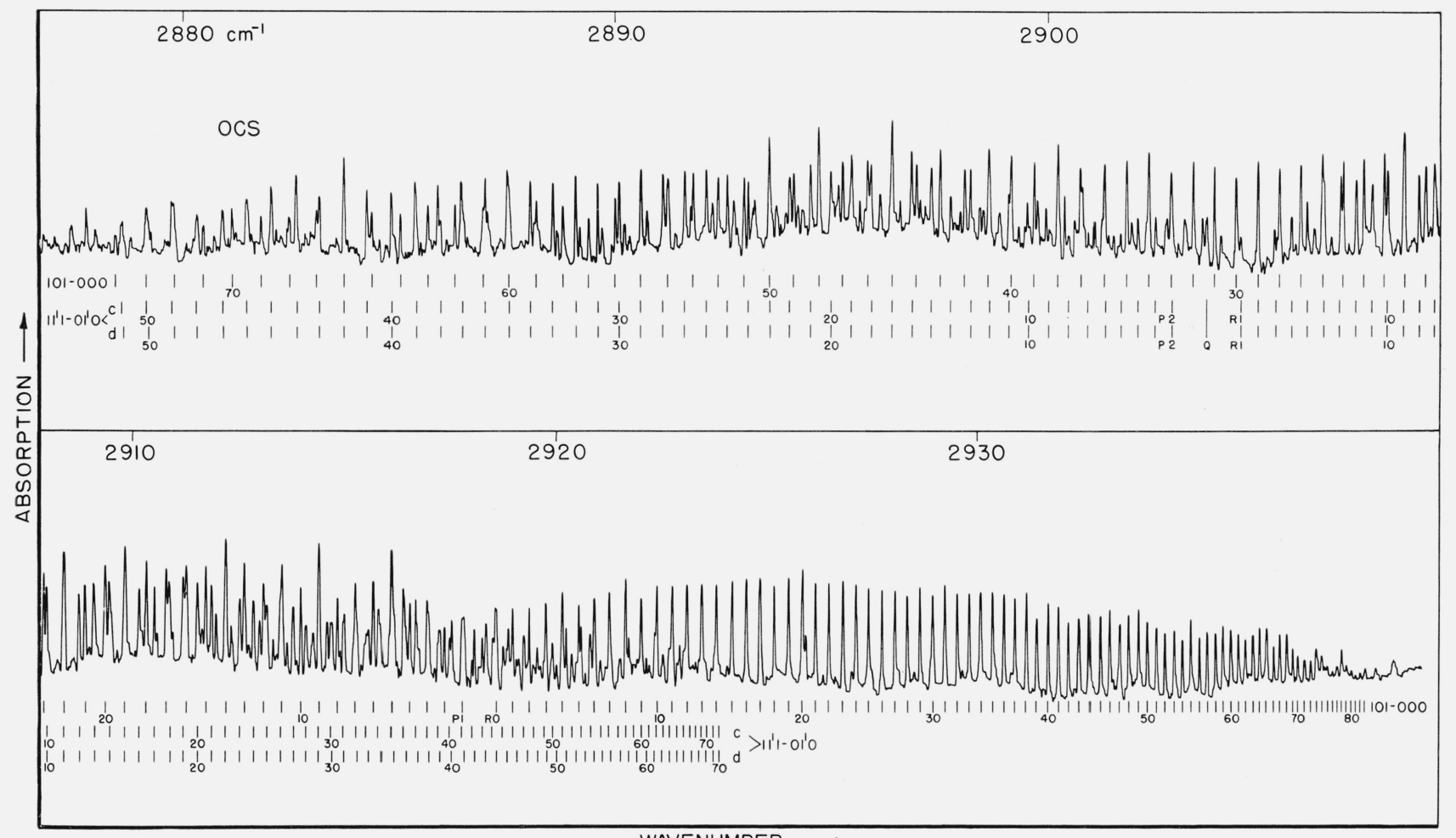

WAVENUMBER

FIGURE 2. The main band and first "hot band" of the $\nu_{1}+\nu_{3}$ transition.

Path length was $8 \mathrm{~m}$ at $1 \mathrm{~mm}$ pressure. 


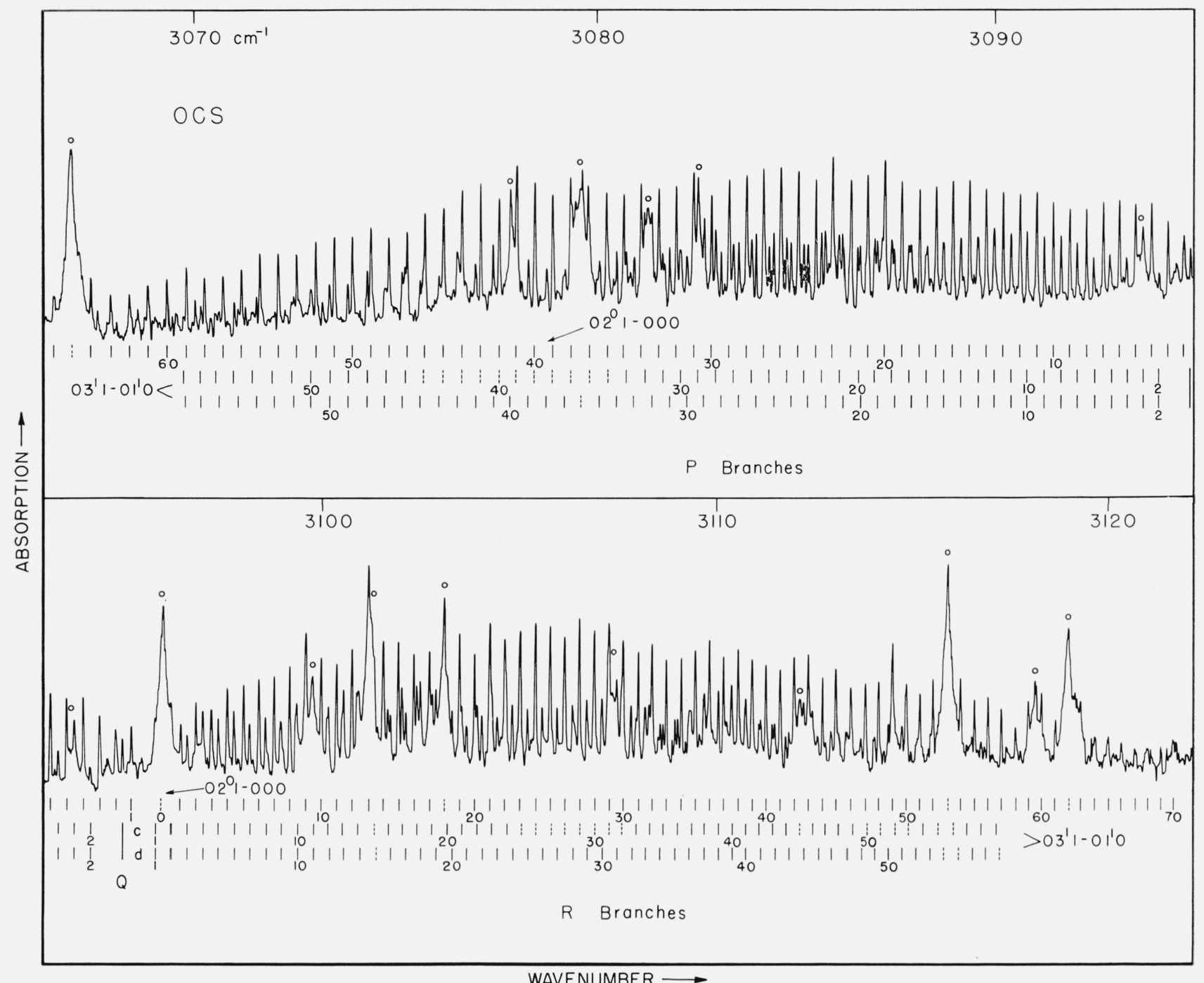

FIgURE 3. Spectrum of OCS from $30 \% 0 \mathrm{~cm}^{-1}$ to $3120 \mathrm{~cm}^{-1}$ obtained at a pressure of $7 \mathrm{~mm}$ and a path length of $8 \mathrm{~m}$.

The circles indicate absorption due to water vapor in the spectrometer.

\section{Rotational Analysis}

The rotational analyses were made using standard methods of the type described by Herzberg [6]. Since very accurate microwave measurements have been made on all the important isotopic species of OCS in the ground vibrational state and in a number of the lower lying vibrational states [7, 8, 9], no attempt was made to obtain accurate determinations of $\mathrm{B}^{\prime \prime}$. Table 1 lists the infrared absorption bands of OCS which have been observed. This table also includes a tabulation of the observed values of $\Delta B$.
In general the values of $\Delta B$ are about what would be estimated for the various bands, but the detailed analysis of the rotational structure gave some $\Delta B$ values larger or smaller than expected. This difference may arise from the bands, especially the "hot bands," overlapping thus making the observed position of a line different from the true position.

The microwave rotational data given in references 7,8 , and 9 have been used wherever applicable to this work.

Apparent differences between measured values of $\alpha$ are sufficiently great to warrant investigation of 
TARLE 1. Infrared spectrum of carbonyl sulfide All frequencies are in wavenumbers

\begin{tabular}{|c|c|c|c|c|c|c|c|c|c|c|}
\hline \multicolumn{3}{|c|}{$\begin{array}{l}\text { Upper } \\
\text { state }\end{array}$} & \multicolumn{3}{|c|}{$\begin{array}{l}\text { Lower } \\
\text { state }\end{array}$} & \multirow{2}{*}{$\begin{array}{c}\text { Freq. }\left(\mathrm{cm}^{-1}\right) \\
\text { a } 520.41\end{array}$} & \multirow[t]{2}{*}{$B^{\prime}-B^{\prime \prime}$} & \multirow[t]{2}{*}{$\left(D^{\prime}-D^{\prime \prime}\right) \times 10^{8}$} & \multirow[t]{2}{*}{$q^{\prime}$} & \multirow[t]{2}{*}{$q^{\prime \prime}$} \\
\hline 0 & $1^{1}$ & & 0 & 0 & 0 & & & & & \\
\hline 1 & & & 0 & 0 & & a 858.954 & & & & \\
\hline 0 & $2^{0}$ & 0 & 0 & 0 & 0 & 1047.1 & & & & \\
\hline 2 & 0 & & 0 & 0 & 0 & b 1711.11 & -0.00124 & & & \\
\hline 1 & $3^{1}$ & 0 & 0 & $1^{1}$ & 0 & 1891.786 & -0.00015 & & & \\
\hline 1 & $2^{0}$ & 0 & 0 & 0 & 0 & 1892. 203 & +0.000128 & -0.41 & & \\
\hline 0 & $1^{1}$ & 1 & 0 & $1^{1}$ & 0 & c 2054.91 & -0.001202 & & & \\
\hline 0 & 0 & 1 & 0 & 0 & 0 & 2062.220 & -0.001219 & +0.08 & & \\
\hline 0 & $4^{0}$ & 0 & 0 & 0 & 0 & 2104.830 & +0.001150 & -0.84 & & \\
\hline 0 & $5^{1}$ & 0 & 0 & $1^{1}$ & 0 & c 2113.93 & +0.00103 & & & \\
\hline 4 & 0 & 0 & 1 & 0 & 0 & 253 & -0.00189 & & & \\
\hline 3 & $1^{1}$ & 0 & 0 & $1^{1}$ & 0 & 2536.691 & -0.001737 & & & \\
\hline 3 & 0 & 0 & 0 & 0 & 0 & 2556. 000 & -0.00186 & -0.4 & & \\
\hline 0 & $2^{2}$ & 1 & 0 & $1^{1}$ & 0 & d 2568.38 & & & & \\
\hline 0 & $1^{1}$ & 1 & 0 & 0 & 0 & 2575. 318 & $\left\{\begin{array}{l}-0.000951 \\
-0.000765\end{array}\right.$ & -0.7 & 0.00019 & \\
\hline 2 & $3^{1}$ & 0 & 0 & $1^{1}$ & 0 & 2724.842 & $\left\{\begin{array}{l}-0.000588 \\
-0.000342\end{array}\right.$ & $\begin{array}{l}\text { 1. } 3 \\
2.0\end{array}$ & 0.00046 & 0.0002 \\
\hline 2 & $2^{0}$ & 0 & 0 & 0 & 0 & 2731.399 & -0.000434 & -0.5 & & \\
\hline 1 & $1^{1}$ & 1 & 0 & $1^{1}$ & 0 & 2903.726 & $\left\{\begin{array}{l}-0.001760 \\
-0.001745\end{array}\right.$ & $\begin{array}{l}-0.8 \\
-1.1\end{array}$ & 0.00025 & 0.000 \\
\hline 2 & 0 & 1 & 1 & 0 & 0 & 2909.511 & -0.00129 & & & \\
\hline 1 & 0 & 1 & 0 & 0 & 0 & 2918.1 & -0.001765 & +0.27 & & \\
\hline 1 & $4^{0}$ & 0 & 0 & 0 & 0 & 2936.756 & +0.000585 & -2.90 & & \\
\hline 0 & $3^{1}$ & 1 & 0 & $1^{1}$ & 0 & 3094.935 & $\left\{\begin{array}{l}-0.000688 \\
-0.000485\end{array}\right.$ & $\begin{array}{l}+0.9 \\
+1.1\end{array}$ & 0.00046 & 0.000 \\
\hline 0 & $2^{v}$ & 1 & 0 & 0 & 0 & 3095.568 & -0.0005348 & -0.36 & & \\
\hline 0 & $6^{0}$ & 0 & 0 & 0 & 0 & 3170. & +0.00157 & & & \\
\hline 0 & 0 & 2 & 1 & 0 & 0 & 3242.439 & -0.001814 & 1. 6 & & \\
\hline 4 & 0 & 0 & e & 0 & 0 & 3393.95 & -0.00249 & & & \\
\hline 2 & 0 & 1 & 0 & 0 & 0 & 3768.48 & -0.00178 & & & \\
\hline 1 & $2^{0}$ & 1 & 0 & 0 & 0 & b 3937.434 & -0.001003 & & & \\
\hline 0 & 11 & 2 & 0 & $1^{1}$ & 0 & 4086. 692 & $\left\{\begin{array}{l}-0.002390 \\
-0.002388\end{array}\right.$ & $\begin{array}{l}1.3 \\
0.3\end{array}$ & & \\
\hline 0 & 0 & 2 & 0 & 0 & 0 & & $\begin{array}{l}-0.002400 \\
-0.002426\end{array}$ & e -0.115 & & \\
\hline 0 & $4^{0}$ & 1 & 0 & 0 & 0 & e 4141.216 & -0.000032 & 2.087 & & \\
\hline 3 & 0 & 1 & 0 & 0 & 0 & e 4609.842 & -0.002998 & 0.034 & & \\
\hline 2 & $2^{0}$ & 1 & 0 & 0 & 0 & e 4773.222 & -0.001493 & 0.260 & & \\
\hline 1 & $1^{1}$ & 2 & 0 & $1^{1}$ & 0 & 4932. 067 & $\left\{\begin{array}{l}-0.002845 \\
-0.002827\end{array}\right.$ & 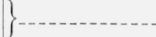 & 0.00032 & 0.000 \\
\hline 1 & 0 & 2 & 0 & 0 & 0 & 4953.876 & -0.002843 & 1.8 & & \\
\hline 2 & 0 & 2 & 0 & 0 & 0 & b 5801.914 & -0.00332 & & & \\
\hline 0 & $1^{1}$ & 3 & 0 & $1^{1}$ & 0 & 6095.47 & -0.00360 & & & \\
\hline 0 & 0 & 3 & 0 & 0 & 0 & e 6117.572 & -0.003618 & -0.335 & & \\
\hline
\end{tabular}

a Obtained by Ritz principle (observed $\nu_{1}=859.0$ ).

b See reference 2 .

d Only $Q$ branch was observed.

e See reference 3 .

the possible effect of the rotation constants quadratic in $v$ (the $\gamma_{i j}$ terms). Enough microwave data is available to allow calculation of some of the $\gamma_{i j}$ terms defined by

$$
\begin{aligned}
B_{\nu_{1} \nu_{2} \nu_{3}}=B_{e}-\sum_{i} \alpha_{i}\left(v_{i}+1 / 2 d_{i}\right) & \\
& +\sum_{i j} \gamma\left(v_{i j}+1 / 2 d_{i}\right)\left(v_{j}+1 / 2 d_{j}\right)
\end{aligned}
$$

where $j \geq i=1,2,3$ and $d_{1}=d_{3}=1, d_{2}=2$. Other $\gamma_{i j}$ terms may be evaluated from infrared values of $\Delta B$. The resulting constants obtained in this way are tabulated in table 2 .

All the $\gamma$ 's which have been found except $\gamma_{13}$ are of the order of magnitude which one would expect. Both values found for $\gamma_{13}$ are almost certainly incorrect. The largest value of $\gamma_{13}$ is obtained from data for the bands $\nu_{1}+\nu_{3}$ and $2 \nu_{2}^{0}+\nu_{3}$. The precision of this data is very good and the deviation is considered to be somewhat greater than might be expected from experimental error. Since the band $\nu_{1}+\nu_{3}$ is only $18.5 \mathrm{~cm}^{-1}$ from the band $\nu_{1}+4 \nu_{2}^{0}$, resonance between these two bands might be responsible for the discrepancy in the rotational constants, even though the coupling parameter would be expected to be small. Another possibility is that the "l-type resonance" described by Amat and Nielsen [9a] may be creating an apparent error in the $\Delta B$ value.
The same situation is found for $\nu_{1}+\nu_{3}$ and $2 \nu_{2}^{0}+\nu_{3}$ of $\mathrm{OC}^{13} \mathrm{~S}$. By using the microwave value of $\alpha_{2}=$ $-0.000336 \mathrm{~cm}^{-1}$ and the infrared value of $\alpha_{3}=$ $0.001142 \mathrm{~cm}^{-1}$ the unperturbed value of $\alpha_{1}$ calculated from the $\Delta B^{\prime}$ 's for $\nu_{1}+\nu_{3}$ and $2 \nu_{2}^{0}+\nu_{3}$ is 0.000540 $\mathrm{cm}^{-1}$. This is smaller than the perturbed value of $\alpha_{1}$ (0.000596) obtained from microwave measurements. This result is, of course, not real and is further evidence that, in both $\mathrm{O}^{16} \mathrm{C}^{12} \mathrm{~S}^{32}$ and $\mathrm{O}^{16} \mathrm{C}^{13} \mathrm{~S}^{32}$, the Fermi diad $\nu_{1}+\nu_{3}$ and $2 \nu_{2}^{0}+\nu_{3}$ may be perturbed'.

The splitting due to $l$-type doubling was observed in four $\pi-\pi$ transitions. The measurements were not extensive enough to determine the exact dependence of the doubling constant $q$ on vibrational quantum number. It is auite evident, however, that $q$ is approximately proportional to $\left(\nu_{2}+1\right) / 2$ as expected.

TABLE 2. Rotational constants for OCS

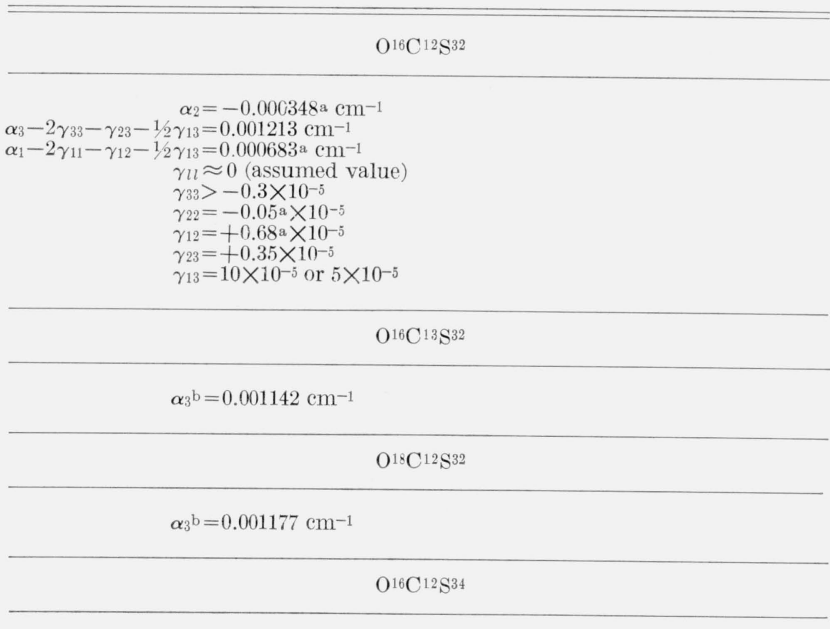

$\alpha_{3} \mathrm{~b}=0.001182 \mathrm{~cm}^{-1}$

a Calculated from microwave frequencies using $c=2.997930 \times 10^{10} \mathrm{~cm} / \mathrm{sec}$. $\mathrm{b}$ These were determined by neglecting the $\gamma_{i j}$ terms.

\section{Vibrational Analysis and Vibrational Constants}

Table 1 contains a list of the absorption bands and constants which have now been measured for OCS. Included in this list are a number of transitions in which the lower state is a vibrationally excited state. By using the following combinations:

$$
\begin{gathered}
(400-000)-(400-100)=3393.95 \\
\quad-2535.00=858.95 \mathrm{~cm}^{-1} \\
(002-000)-(002-100)=4101.387 \\
-3242.439=858.948 \mathrm{~cm}^{-1} \\
(201-000)-(201-100)=3768.48 \\
\quad-2909.511=858.97 \mathrm{~cm}^{-1}
\end{gathered}
$$

a weighted average value of $858.954 \mathrm{~cm}^{-1}$ is found for $\nu_{1}$. This is in very good agreement with a value of 859.03 which we have measured on a small commercial grating spectrometer. The value of $\nu_{2}$ is determined to be $520.41 \mathrm{~cm}^{-1}$ by using $\left(01^{1} 1-000\right)$ 
- $\left(01^{1} 1-01^{1} 0\right)$. This may be compared with the value of 521.5 found by Bartunek and Barker [10]. The $2 \nu_{2}^{0}$ band has also been measured in this laboratory on a small commercial grating spectrometer and the band center was found to be $1047.1 \mathrm{~cm}^{-1}$. The rotational structure of this band was rather poorly resolved so that the band center could only be determined with certainty within one value of $J$.

Seven absorption bands have also been measured and assigned to isotopic species other than $\mathrm{O}^{16} \mathrm{C}^{12} \mathrm{~S}^{32}$. These are given in table 3. More absorption bands have been found for $\mathrm{OC}^{13} \mathrm{~S}$ than for $\mathrm{OCS}^{34}$, although the abundance ratio is $1: 4$. This is undoubtedly due to the greater shift in absorption frequencies for $\mathrm{OC}^{13} \mathrm{~S}$

TABLE 3. Observed infrared bands of isotopic OCS

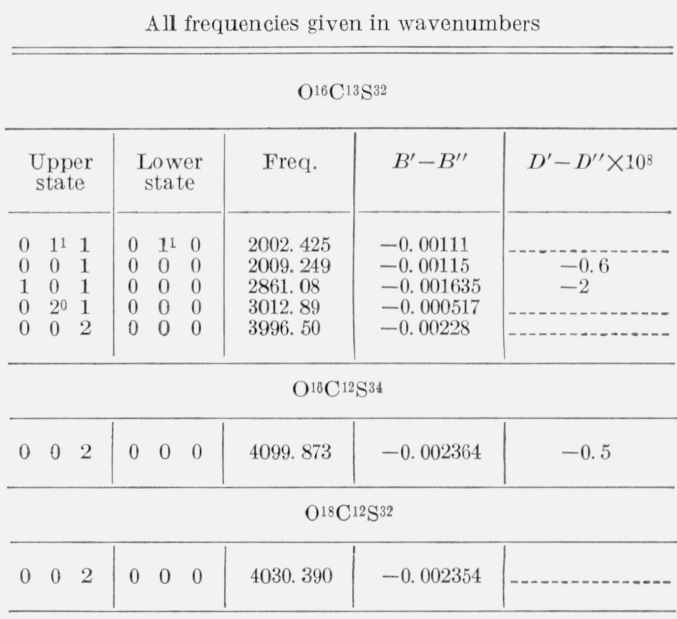

With the large amount of vibrational data now available, it is possible to calculate some of the quadratic vibrational constants by two or more independent methods. Upon neglecting the cubic terms $\left(y_{i j k}\right)$ three different values are found for the combination $x_{11}+4 x_{22}+x_{12}$. These are $-5.37,-5.01$, and $-3.63 \mathrm{~cm}^{-1}$. The disagreement indicates that either the cubic terms must be taken into account, or else an unexpected perturbation is present. For the series $\nu_{3}, 2 \nu_{3}, 3 \nu_{3}$ it has previously been shown
[2] that only a very small cubic term is required to give nearly perfect agreement between calculated and observed frequencies. This is also the case for the "hot bands" of these transitions. From these bands the pertinent cubic constants are found to be $y_{233}=-0.03 \mathrm{~cm}^{-1}$ and $y_{333}=+0.01 \mathrm{~cm}^{-1}$. Both of these are of the expected order of magnitude. These are the only cubic terms which can be determined explicitly from the available data without determining the unperturbed frequencies of the resonating vibrations.

One can also evaluate various combinations of quadratic and cubic constants by suitable combinations of the sums of various Fermi resonant multiplets but the resulting values are of dubious accuracy. One such combination yields $y_{113}+4 y_{233}+y_{123}=1.38$ $\mathrm{cm}^{-1}$ which seems rather large for such a heavy molecule.

Because of inconsistencies in the values of the $\Delta B$ 's for the Fermi resonant bands, it seems premature at this time to find the unperturbed frequencies of these vibrations. Further very careful work must be done on OCS in order to ascertain whether any further perturbations are present.

\section{References}

[1] H. J. Callomon and H. W. Thompson, Proc. Roy. Soc. A2\%2, 431 (1954).

[2] H. C. Allen, E. K. Plyler, and L. R. Blaine, J. Chem. Phys. 26, 400 (1957)

[3] G. D. Saksena, T. A. Wiggins, and D. H. Rank, J. Chem. Phys. 31, 839 (1959).

[4] E. K. Plyler and L. R. Blaine, J. Research NBS 62, 7 (1959).

[5] E. K. Plyler, L. R. Blaine, and E. D. Tidwell, J. Research NBS 55, 183 (1955)

[6] G. Herzberg, Infrared and Raman spectra of polyatomic molecules (D. Van Nostrand Co., Inc., New York, 1954).

[7] C. H. Townes and A. H. Schawlow, Microwave Spectroscopy (McGraw-Hill Book Co., Inc., New York, 1955).

[8] W. C. King and W. Gordy, Phys. Rev. 93, 407 (1954).

[9] M. Peter and M. W. P. Strandberg, Phys. Rev. 95, 622 (1954).

[9a] G. Amat and H. H. Nielsen, J. Mol. Spectroscopy 2, 163 (1958).

[10] P. Bartunek and E. F. Barker, Phys. Rev. 48, 516 (1935).

(Paper 66A2-147) 